This is the accepted manuscript made available via CHORUS. The article has been published as:

\title{
Picosecond Electric-Field-Induced Threshold Switching in Phase-Change Materials
}

Peter Zalden, Michael J. Shu, Frank Chen, Xiaoxi Wu, Yi Zhu, Haidan Wen, Scott Johnston, Zhi-Xun Shen, Patrick Landreman, Mark Brongersma, Scott W. Fong, H.-S. Philip Wong, Meng-Ju Sher, Peter Jost, Matthias Kaes, Martin Salinga, Alexander von Hoegen, Matthias Wuttig, and Aaron M. Lindenberg

Phys. Rev. Lett. 117, 067601 - Published 5 August 2016

DOI: 10.1103/PhysRevLett.117.067601 


\section{Picosecond electric-field-induced threshold switching in phase-change materials}

(Peter Zalden ${ }^{+1,2}$, Michael J. Shu $\left.{ }^{1,3}\right)^{*}$, Frank Chen ${ }^{1,4}$, Xiaoxi Wu ${ }^{1}$, Yi Zhu ${ }^{5}$, Haidan Wen ${ }^{5}$, Scott Johnston $^{3}$, Zhi-Xun Shen ${ }^{3}$, Patrick Landreman ${ }^{8}$, Mark Brongersma ${ }^{8}$, Scott W. Fong ${ }^{4}$, H.-S.Philip Wong ${ }^{4}$, Meng-Ju Sher ${ }^{8}$, Peter Jost ${ }^{6}$, Matthias Kaes ${ }^{6}$, Martin Salinga ${ }^{6}$, Alexander von Hoegen ${ }^{6}$, Matthias Wuttig ${ }^{6,7}$ and Aaron Lindenberg ${ }^{+1,2,8}$

* These authors contributed equally

+ Corresponding authors: Peter Zalden, peter@,zalden.de; Aaron Lindenberg, aaronl@stanford.edu

1) Stanford Institute for Materials and Energy Sciences, SLAC National Accelerator Laboratory, Menlo Park, CA 94025, USA

2) PULSE Institute, SLAC National Accelerator Laboratory, Menlo Park, CA 94025, USA

3) Department of Applied Physics, Stanford University, Stanford, CA 94305, USA

4) Department of Electrical Engineering, Stanford University, Stanford, CA 94305, USA

5) Advanced Photon Source, Argonne National Laboratory, Argonne, IL 60439, USA

6) I. Physikalisches Institut (IA), RWTH Aachen University, 52056 Aachen, Germany

7) JARA - Fundamentals of Information Technology, RWTH Aachen University, 52056 Aachen, Germany

8) Department of Materials Science and Engineering, Stanford University, Stanford CA 94305, USA

Many chalcogenide glasses undergo a breakdown in electronic resistance above a critical field strength. Known as threshold switching, this mechanism enables field-induced crystallization in emerging phasechange memory. Purely electronic as well as crystal nucleation assisted models have been employed to explain the electronic breakdown. Here, picosecond electric pulses are used to excite amorphous $\mathrm{Ag}_{4} \mathrm{In}_{3} \mathrm{Sb}_{67} \mathrm{Te}_{26}$. Field-dependent reversible changes in conductivity and pulse-driven crystallization are observed. The present results show that threshold switching can take place within the electric pulse on sub-picosecond time-scales - faster than crystals can nucleate. This supports purely electronic models of threshold switching and reveals potential applications as an ultrafast electronic switch.

Threshold switching is an essential mechanism in the application of phase-change materials (PCM), where electric pulses are used to thermally cycle an active material between its amorphous and crystalline states[1]-[3]. The amorphous phase of most chalcogenide glasses employed in this emerging technology has a conductivity that is too low to enable Joule heating to the crystallization temperature using voltages that are readily available in an electronic device[4]. However, the so-called "threshold switching" (TS) induces a breakdown in electronic resistance - for instance by three orders of magnitude[5] in the common $\mathrm{PCM} \mathrm{Ge} \mathrm{Sb}_{2} \mathrm{Te}_{5}$. This allows for significant heating leading to crystallization if the electric current is maintained for a sufficient duration. On the other hand, if the electric field is removed quickly after TS, the material returns to its amorphous, highly resistant state[6]. These observations have led to the development of a collection of different models to explain this effect[3], [7], [8]. In particular the influence of atomic rearrangements has been discussed ever since the first experimental finding of TS, with early attempts of explaining it based on field-driven nucleation effects[9]. It was also shown that TS can be explained based on entirely electronic models, coupled to the atomic temperature[5][8]. The underlying electronic transport mechanism in amorphous PCMs requires 
several models to explain its field dependence. At lowest field strength, Ohmic behavior is observed, whereas upon increasing field strength, the current begins to depend exponentially on the field with a sequence of Poole, Poole-Frenkel and Thermally Assisted Tunneling transport. At even higher field strength, direct tunneling of carriers becomes possible[10].

Early studies of TS in memory cells revealed the existence of a critical threshold field and a delay time between the application of an electric field and the breakdown in resistivity[11], [6]. It was found that this delay time decreases exponentially with increasing voltage - for delay times in the sub- $\mu \mathrm{s}$ regime. More recent data extend the measurement range to higher voltages[8], [12] and indicate that the delay may converge toward a lower limit on the order of few nanoseconds. This would imply an upper limit in the electronically driven operation speed of phase-change memory devices. So far the shortest pulses reported for electrical excitation of a phase-change memory device based on $\mathrm{Ge}_{2} \mathrm{Sb}_{2} \mathrm{Te}_{5}$ (GST) are of $0.5 \mathrm{~ns}$ duration[13]. Here we use few picosecond single-cycle electromagnetic waves with $0.5 \mathrm{THz}$ center frequency to optically apply an electric field to the PCM, as shown in Fig. 1a. The impact of these pulses in a subthreshold regime, where no irreversible change occurs, has been described in earlier work[14]. To enhance the electric field strength we use electrode patterns with sub-wavelength, $1 \mu \mathrm{m}$ gaps (see schematic drawing in Fig. 1b)[15]. Samples consist of $100 \mathrm{~nm}$-thick gold bars in an interdigitated pattern, with $1 \mu \mathrm{m}$ gap width and $10 \mu \mathrm{m}$ electrode width, thermally evaporated and lithographically patterned on a quartz substrate. A $390 \mathrm{~nm}$ thick film of $\mathrm{Ag}_{4} \mathrm{In}_{3} \mathrm{Sb}_{67} \mathrm{Te}_{26}$ (AIST) is deposited by DC magnetron sputter deposition on top of this structure, filling the gaps between the gold bars. The film is capped with $20 \mathrm{~nm}$ of alumina grown using atomic layer deposition (ALD). The main advantage of AIST over GST for this study is its lower threshold field strength of $190 \mathrm{kV} / \mathrm{cm}$ as compared to $560 \mathrm{kV} / \mathrm{cm}$ for GST[16], while AIST is equally known to crystallize rapidly[17][18].

Incoming $\mathrm{THz}$ pulses interact with the linear electrodes enhancing the electric field inside the PCM as compared to a bare film[19]. For the given geometry with gaps of $1 \mu \mathrm{m}$ the field is enhanced by a factor of 6.8 as compared to the field strength in AIST in the absence of electrodes - as verified experimentally and confirmed by finite element simulations presented in the methods section of the supplementary material[20]. With these gaps our experimental setup allows applying THz pulses with peak field strength of up to $480 \pm 90 \mathrm{kV} / \mathrm{cm}$ to the PCM, based on the incident field $100 \mathrm{kV} / \mathrm{cm}$ and the field enhancement accounting for reflection losses. Each $\mathrm{THz}$ pulse has a duration of only few picoseconds - orders of magnitude shorter than the delay times reported in literature. Each pulse drives a current that Joule heats the PCM by the temperature $\Delta T$,

$$
\Delta T=\int \sigma(E(t)) \cdot E(t)^{2} / c_{p} \mathrm{dt}
$$

for THz conductivity $\sigma$, specific heat $c_{p}$, and enhanced THz electric field $E(t)$ depicted in Fig. 1a for the incoming pulse. Note that this is equivalent to absorption of THz photons by the PCM with imaginary component of dielectric function $\varepsilon^{\prime \prime}=\sigma \cdot\left(\varepsilon_{0} \omega\right)^{-1}$. With $\sigma=2.5 \mathrm{~S} / \mathrm{cm}$ for amorphous AIST at THz frequencies[21], one concludes that every excitation pulse of $480 \mathrm{kV} / \mathrm{cm}$ heats the AIST by $0.6 \mathrm{~K}$. At 
room temperature, the heating due to a single pulse is far below what is required to reach the crystallization temperature of $440 \mathrm{~K}[22]$.

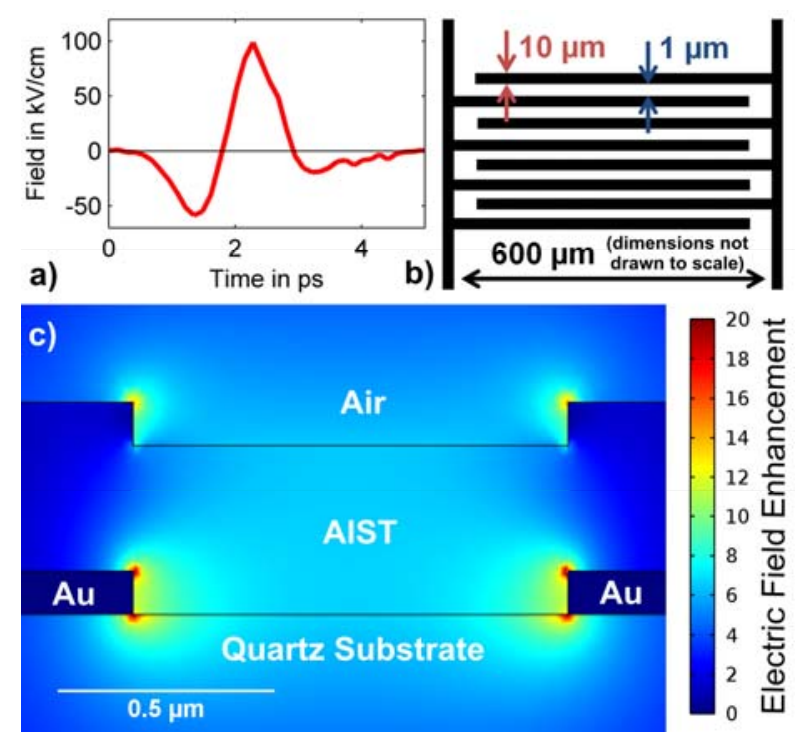

Fig. 1: THz pulses with peak field strength of up to $100 \mathrm{kV} / \mathrm{cm}$ and picosecond duration (a) interact with an array of electrodes (b), whose gaps are filled with the PCM AIST. The electrodes enhance the electric field (c) over that observed in a bare film (capping layer not included).

We expose the device to $\mathrm{THz}$ pulses of $480 \mathrm{kV} / \mathrm{cm}$ peak field strength at a repetition rate of $30 \mathrm{~Hz}$ for 30 seconds. Even though the temperature of AIST is not expected to rise by more than $0.6 \mathrm{~K}$ during repetitive excitation, we observe crystallized filaments that were formed during the exposure. Crystallized material is observed by optical white light microscopy after $\mathrm{THz}$ exposure via the formation of highly reflective spots that did not exist in the unexposed sample (Fig. 2a)[23]. These spots also possess a higher electronic conductivity, as demonstrated by microwave impedance microscopy (MIM) data shown in Fig. 2b. The higher conductivity of the crystallized material produces a decrease in the depicted in-phase component of the reflected microwave signal. Finally, we present $\mathrm{x}$-ray diffraction data with micrometer spatial resolution (Fig. 2c\&d), which evidence the occurrence of Bragg diffraction characteristic of crystalline AIST at specific $\mu \mathrm{m}$-sized positions within the gap. Fig. $2 \mathrm{c}$ maps the integrated diffracted intensity from gold around $q=2.67 \AA^{-1}[24]$ and reveals the end of one of the gold electrodes. Mapping the same area based on the integrated diffraction from crystalline AIST around $q=$ $2.02 \AA^{-1}[17],[22]$ results in Fig. 2d, which demonstrates a $\mu \mathrm{m}$-sized area of polycrystalline AIST at the corner of the electrode. This is not a single grain, as evidenced by the occurrence of multiple diffraction spots with the same $q$ (not shown). In this image, the crystalline filament appears elongated along the slit due to the elliptical x-ray footprint on the sample of $5 \mu \mathrm{m}$ long diameter (350 $\mathrm{nm}$ vertical diameter). These property changes in optical reflectivity, electronic conductivity and lattice ordering evidence crystallization after repetitive $\mathrm{THz}$ excitation. From the fact that crystallization occurs, we can derive 
that crystallization temperatures were reached. This implies, following eq. 1, that the electronic conductivity must increase by more than two orders of magnitude during the application of the $\mathrm{THz}$ pulse. Our results therefore show that TS takes place on the picosecond timescale of the THz pulse. The field strength used in this study is comparable to that used for switching electronic devices and the electronic transport should therefore follow the same fundamental principles.

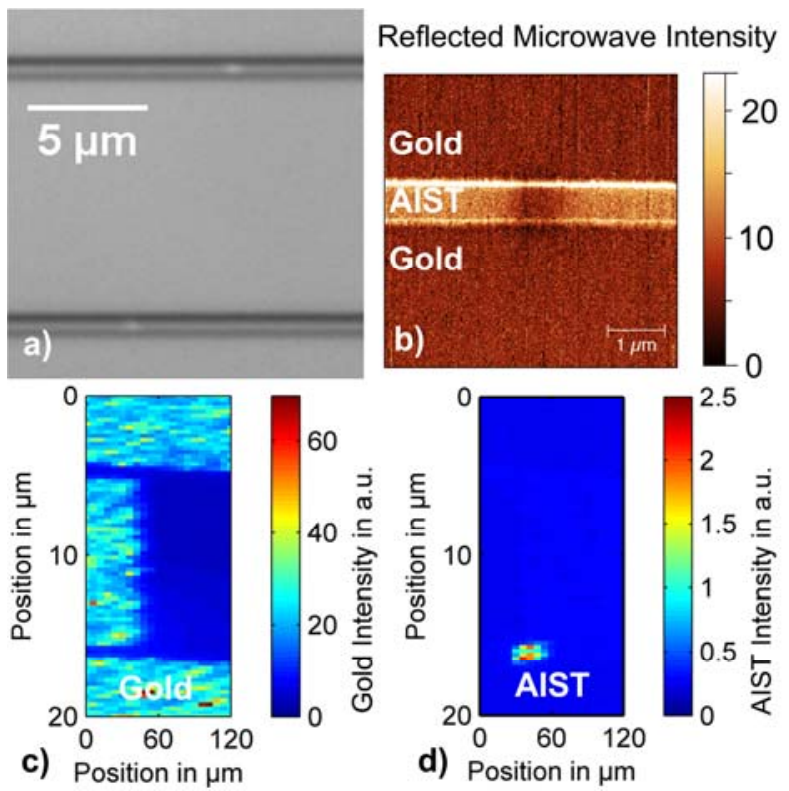

Fig. 2: Evidence for filamentary crystallization of AIST after repetitive THz exposure (ex-situ): a) Mapping of optical white light reflectivity reveals brighter marks within the gap, b) Microwave dissipation (in-phase) signal shows a local decrease in resistance within the slit, c)+d) X-ray diffraction intensity in specific q-ranges for gold c) and AIST d), respectively, reveals local lattice ordering.

In order to investigate the THz-induced crystallization mechanism in more detail, we have performed a THz pump/near-infrared (NIR) probe measurement of the magnitude of the THz-induced Joule heating. This measurement was carried out on similar samples, with split-ring resonators (SRR) in place of the linear electrodes. These resonator structures are depicted schematically in the inset of Fig. 3b). They confine the field to act on a well-defined area of AIST within the slit that can be probed using a microscopically focused near-infrared (NIR) pulsed beam. Fig. 3a) shows the result of a time delay scan upon repetitive THz excitation in a pump probe scheme. The electric field modulates the NIR transmission via an instantaneous electroabsorption effect while driving a current that Joule heats the material. After the excitation at about 2 ps delay, a fast relaxation mechanism is observed with time constant of $3 \pm 1$ ps - most likely due to the relaxation of emitted carriers. Following the relaxation, the transmission change remains constant over at least tens of picoseconds until the heat diffuses out of the PCM. We scale this transmission change to a calibrated temperature dependent transmittance (see supplementary Fig. S4[20]) such that the NIR transmission 8 ps after THz excitation is a measure of the 
temperature jump in the AIST. The resulting temperature jump is shown in Fig. 3b), measured repetitively while cycling the $\mathrm{THz}$ pulse fluence to a level just below the onset of crystallization. These data reveal that $\mathrm{THz}$ pulses increase the temperature by tens of Kelvin. Note that the experiment determines an average temperature over the $8 \mu \mathrm{m}$ diameter of the probe beam, whereas Fig. 2 shows that only few $\mu \mathrm{m}$-sized conducting channels are formed inside the PCM. Joule heating is significantly higher in these filaments than in the surrounding material, and therefore the experimentally obtained temperature underestimates the peak temperature of the filament. Considering this, we can estimate the temperature of the switched filament by scaling the experimental temperature jump by the ratio of the probe beam diameter to the conducting channel size. Based on the $>15 \mathrm{~K}$ temperature jumps observed and the formation of $1 \mu \mathrm{m}$ wide filaments along the $8 \mu \mathrm{m}$ beam diameter, this gives temperature jumps of $\sim 120 \mathrm{~K}$ comparable to the crystallization temperature in this just-below-crystallization regime.

Therefore, our experimental data are consistent with a Joule heating-based thermal model of nucleation and growth to explain crystallization. It is worth mentioning that the active volume in our devices is well above the lower limit of percolation theory[25].

The data in Fig. 3b) also reveal that upon exceeding a specific threshold field, whose absolute value will be discussed later, the temperature jump starts to increase more dramatically, with a strongly non-linear dependence on the applied electric field. Since the $\mathrm{THz}$ fluence is proportional to the square of the peak field, it is evident from eq. 1 that this factor of ten increase in slope (heating/fluence) implies that the conductivity of the probed material increases by at least an order of magnitude to $>25 \mathrm{~S} / \mathrm{cm}$ when approaching crystallization conditions. Commonly, threshold switching is accompanied by at least three orders of magnitude increase in conductivity over the DC value of $0.025 \mathrm{~S} / \mathrm{cm}$. In the present case the increase appears smaller for two reasons. Firstly, an additional phonon absorption mechanism of 2.5 $\mathrm{S} / \mathrm{cm}$ conductivity is present at $\mathrm{THz}$ frequencies[21], acting as a field-independent background. The effect of this constant phonon contribution is depicted as a red line in Fig. 3b), obtained from fitting the constant conductivity data at lowest $\mathrm{THz}$ fluence. Further experimental evidence for this low-field $\mathrm{THz}$ conductivity/absorption mechanism of amorphous AIST can be found in part D of the supplementary information[20]. Secondly, the probe beam averages over inhomogeneously switched material, reducing the derived modulation in the conductivity of the switched filament and implying conductivities of order $250 \mathrm{~S} / \mathrm{cm}$ inside the filament. Therefore, our data provide evidence for a THz-induced increase of the electronic conductivity by more than three orders of magnitude over the DC value - just as commonly observed during TS. This increase in electronic conductivity is larger than the field dependence of the sub-threshold conductivity in AIST[10] and therefore confirms that an electronic instability occurs during the picosecond $\mathrm{THz}$ excitation. 

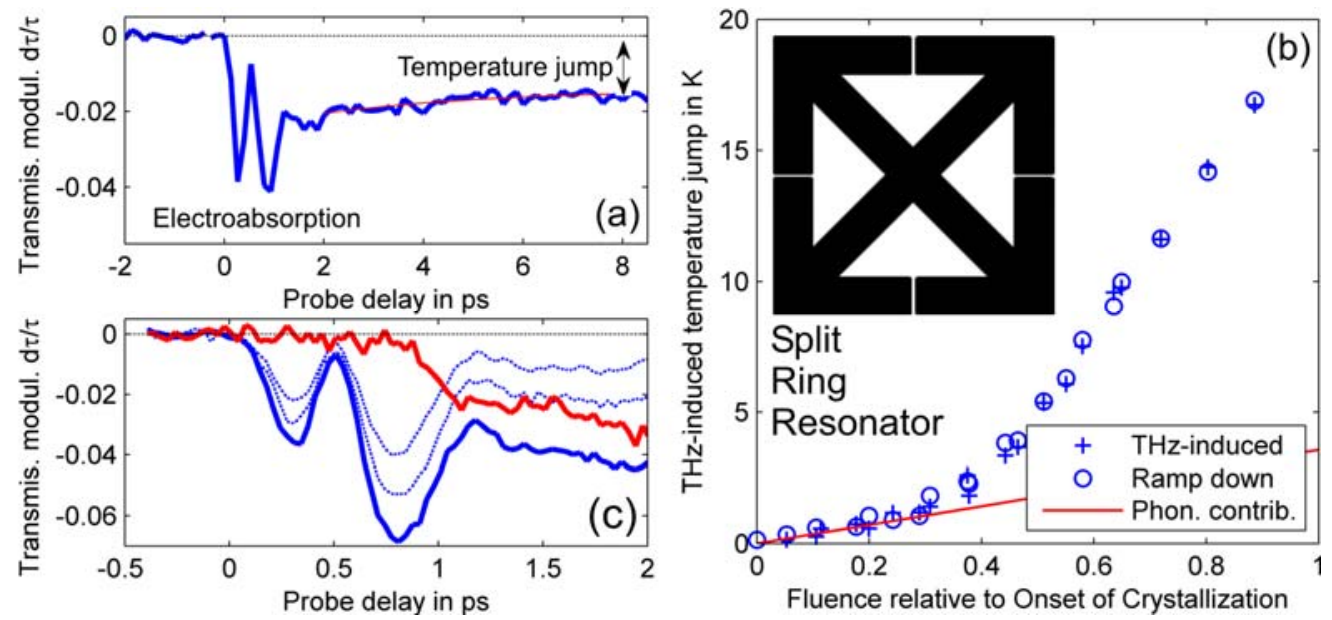

Fig. 3: (a) THz pulses are absorbed in the PCM and induce temperature jumps that modulate its optical transmission after carrier relaxation (blue curve with red refined exponential decay). This long-lived transmission change is due to Joule heating of the PCM. (b) At low THz fluences this temperature jump also scales linearly with the square of the electric field, dominated by a phonon-absorption mechanism. At higher peak fields, however, a one order of magnitude increase in slope (conductivity) marks the onset of TS. (c) The instantaneous field-induced modulation due to electroabsorption scales linearly with the square of the electric field (intensity) and can be subtracted from the data at highest fluence (thick curve), revealing the ultrafast onset of the non-linear Joule heating mechanism (red curve).

Another characteristic feature of TS is the reversibility below the onset of crystallization. Fig. 3b) also provides evidence for this reversibility, because the data points were obtained in a pumpprobe experiment and therefore TS takes place during single pulse excitation. It is worth mentioning that the data in Fig. 3b) cannot be explained by gradual crystallization of the PCM, because the initial lowconductivity regime is retained once the peak field is decreased again. Furthermore, at stronger fields when the device crystallizes, the formation of a permanent crystalline conduction path suppresses the field enhancement of the SRR, which is seen experimentally as an immediate weakening of the pumpprobe effect (not shown).

The timescale of the THz-induced transmission change (Fig. 3a) is hidden underneath the electroabsorption signal, which scales with the square of the electric field[14][26] and dominates at weak excitation conditions. Fig. 3c) shows delay scans at three different $\mathrm{THz}$ fluences (blue curves) of 0.4, 0.55 and 0.7 times the fluence at the onset of crystallization. Based on these data it becomes possible to subtract the electroabsorption effect, revealing the ultrafast heating mechanism in the remaining signal (red curve), previously hidden in the raw data (thick blue curve). This scan reveals the electronic breakdown mechanism associated with TS on a sub-picosecond timescale.

It is well known that the electric field required for TS increases for short pulse durations[11], [26]. With the SRR structures, the confinement of the electric field precludes an experimental 
determination. Therefore, we performed the same pump-probe experiment on the linear electrode structures discussed earlier, where the absolute field strength can be simulated and measured for a given $\mathrm{THz}$ fluence based on the sub-threshold homogeneous heating of the phase-change material. We find that the absolute threshold field for AIST is $230 \pm 60 \mathrm{kV} / \mathrm{cm}$ - not significantly deviating from literature data using DC pulses $(190 \mathrm{kV} / \mathrm{cm})$. Details of this determination can be found in part B of the supplementary information[20]. It is worth mentioning that the literature value was obtained with unipolar pulses, whereas for bipolar THz pulses the 1.5 times higher peak-to-peak field swing may be relevant for inducing TS. Presently, no comparative study of AC vs. DC threshold field strengths is known to the authors. At peak field strength of $340 \mathrm{kV} / \mathrm{cm}$ and above, crystallization is found to take place in the linear electrodes. This ratio of threshold fluence to onset of crystallization $(230 / 340)^{2}=0.46$ is very similar to the ratio found for the SRR devices (see Fig. 3b).

A summary of models to describe TS is beyond the scope of this manuscript and therefore we will compare our results to a very recent and comprehensive model[10]. It is based on a combination of linear and non-linear electronic transport mechanisms, which are dominating depending on the applied field strength. Extrapolating this quantitative model for AIST including direct tunneling to ambient temperature of $300 \mathrm{~K}$, one obtains a total density of emitted carriers on the order $5 * 10^{20} \mathrm{~cm}^{-3}$ for a THz waveform $\mathrm{E}(\mathrm{t})$ of $360 \mathrm{kV} / \mathrm{cm}$ peak field strength. This value is based on an assumed mobility of $1 \mathrm{~cm}^{2}$ $(\mathrm{Vs})^{-1}$ and a high-field slope $\mathrm{F}_{\mathrm{c}}$ of $9.5 \pm 0.5 \mathrm{~V} / \mu \mathrm{m}$, whose uncertainty dominates that of the resulting carrier concentration. The absolute conductivity resulting from this estimate is on the order $100 \mathrm{~S} / \mathrm{cm}$, in good agreement with the present results.

One alternative model proposed to explain TS relies on a field-driven nucleation mechanism, where the electric field induces the transient formation of subcritical crystalline nuclei with orders of magnitude higher conductivity[9], [27]. Based on our findings we can rule out models for TS that are based on nucleation processes, because we show that the sub-picosecond timescale of TS is shorter than what is required for nucleation of crystalline material: The nucleation rate is limited by the rate at which atoms vibrate and attempt to nucleate. In case of AIST, within a picosecond only less than five atomic vibrations are possible for each atom[28], which is insufficient to establish the periodic arrangement of several atoms in a crystal. Therefore, TS must be of solely electronic origin. In such a model, the lower limit for the time delay associated with TS becomes negligible as compared to the nanosecond thermal crystallization mechanism if sufficiently high field strengths are employed. Consequently, this minimum delay associated with TS is expected to be significantly shorter than a picosecond.

To support the consistency of the overall mechanism, we briefly discuss its energetics based on the sample with linear electrodes. At the onset of crystallization, each $\mathrm{THz}$ pulse induces a field strength of $340 \mathrm{kV} / \mathrm{cm}$ inside AIST. This value is obtained, taking into account the full dielectric functions of all materials involved. Simulations also show that this field is not diminished in the presence of narrow conducting filaments with conductivity of order $10^{3} \mathrm{~S} / \mathrm{cm}$, comparable to the conductivity of the crystalline phase (see supplementary material, Fig. S6[20]). Thus energy is effectively channeled through 
the electrodes toward the conductive filament. Note that this high conductivity, required for heating to the crystallization temperature, occurs at an applied field significantly above the TS voltage as seen in Fig. 3b). These filaments absorb an energy density $\sigma E^{2} t=150 \mathrm{~J} \mathrm{~cm}^{-3}$, enough to heat the material to the crystallization temperature, given the specific heat of AIST of $1.27 \mathrm{~J} \mathrm{~cm}^{-3} \mathrm{~K}^{-1}$ [28]. In order to heat one filament of $1 \mu \mathrm{m}$ width to the crystallization temperature, this requires absorption of $70 \mathrm{pJ}$ of energy, much less than the $45 \mathrm{~nJ}$ of THz energy incident on the area of each slit and half of both its neighboring electrode bars. Therefore, we conclude that a model based on purely thermal crystallization can explain the experimental results.

In conclusion, we have demonstrated that an amorphous PCM crystallizes under repetitive excitation with single-cycle $\mathrm{THz}$ pulses of few picosecond duration and sufficient field strength. We provide experimental evidence for most of the characteristic features of threshold switching: The formation of conducting filaments through highly non-linear conduction mechanisms, a threshold field that is in reasonable agreement with literature values and the reversibility at above threshold, but subcrystallization conditions. In the initially amorphous device, THz pulses induce TS during the picosecond excitation. TS increases the conductivity and the resulting higher current heats the material locally above the crystallization temperature. Upon repetitive excitation, atomic rearrangements toward the crystalline phase can take place. Due to the thermodynamically irreversible nature of crystallization, these modifications are retained after the material has cooled down by heat transport into the substrate[29], [30]. The observation of picosecond TS is particularly important for ovonic switches, sometimes used as pre-selectors in memory devices[31], which then allow sub-picosecond access to the specific memory cell. In this sense, TS allows the design of ultrafast electronic switches.

Research was supported by the U.S. Department of Energy, Basic Energy Sciences, Materials Sciences and Engineering Division. MW gratefully acknowledges support by the Deutsche Forschungsgemeinschaft through SFB 917. PZ gratefully acknowledges funding from the Alexander von Humboldt Foundation. The use of the Advanced Photon Source was supported by the U.S Department of Energy, Office of Science, Office of Basic Energy Sciences, under Contract No. DE-AC02-06CH11357. MS and MK acknowledge funding from the DIASPORA project of the FP7-IAPP Marie-Curie Action by the European Commission. SWF and HSPW are supported in part by the Stanford Non-Volatile Memory Technology Research Initiative (NMTRI). The MIM work was supported by NSF DMR 1305731.

[1] H. P. Wong, S. Raoux, S. Kim, J. Liang, J. P. Reifenberg, B. Rajendran, M. Asheghi, and K. E. Goodson, "Phase Change Memory," Proc. IEEE, vol. 98, no. 12, pp. 2201-2227, Dec. 2010.

[2] M. Wuttig and N. Yamada, "Phase-change materials for rewriteable data storage," Nat. Mater., vol. 6, no. 11, pp. 824-832, 2007.

[3] D. Ielmini and Y. Zhang, "Analytical model for subthreshold conduction and threshold switching in chalcogenide-based memory devices,” J. Appl. Phys., vol. 102, no. 5, p. 054517, 2007.

[4] G. W. Burr, M. J. Breitwisch, M. Franceschini, D. Garetto, K. Gopalakrishnan, B. Jackson, C. Lam, and A. Luis, "Phase change memory technology," J. Vac. Sci. Technol. B, vol. 28, no. 2, pp. 223-262, 2010.

[5] A. Redaelli, A. Pirovano, F. Pellizzer, A. L. Lacaita, D. Ielmini, and R. Bez, "Electronic switching effect and phase-change transition in chalcogenide materials," IEEE Electron Device Lett., vol. 25, no. 10, pp. 684-686, 2004. 
[6] A. E. Owen and J. M. Robertson, "Electronic conduction and switching in chalcogenide glasses," IEEE Trans. Electron Devices, vol. 20, no. 2, p. 105, 1973.

[7] M. Nardone, M. Simon, I. V. Karpov, and V. G. Karpov, "Electrical conduction in chalcogenide glasses of phase change memory," J. Appl. Phys., vol. 112, no. 7, p. 071101, 2012.

[8] M. Le Gallo, A. Athmanathan, D. Krebs, and A. Sebastian, "Evidence for thermally assisted threshold switching behavior in nanoscale phase-change memory cells," J. Appl. Phys., vol. 119, no. 2, p. 025704 , 2016.

[9] S. R. Ovshinsky, "Reversible Electrical Switching Phenomena In Disordered Structures," Phys. Rev. Lett., vol. 21, no. 20, p. 1450, 1968.

[10] M. Kaes, M. Le Gallo, A. Sebastian, M. Salinga, and D. Krebs, "High-field electrical transport in amorphous phase-change materials,” J. Appl. Phys., vol. 118, p. 135707, 2015.

[11] S. R. Ovshinsky, "Reversible electrical switching phenomena in disordered structures," Phys. Rev. Lett., vol. 21, no. 20, p. 1450, 1968.

[12] M. Wimmer and M. Salinga, "The gradual nature of threshold switching," New J. Phys., vol. 16, no. 11, p. 113044, Nov. 2014.

[13] D. Loke, T. H. Lee, W. J. Wang, L. P. Shi, R. Zhao, Y. C. Yeo, T. C. Chong, and S. R. Elliott, "Breaking the Speed Limits of Phase-Change Memory," Science, vol. 336, no. 6088, pp. 1566-1569, 2012.

[14] M. Shu, P. Zalden, F. Chen, B. Weems, I. Chatzakis, F. Xiong, R. Jeyasingh, M. Hoffmann, E. Pop, P. Wong, M. Wuttig, and A. Lindenberg, "Ultrafast electric-field-induced processes in GeSbTe phase-change materials," Appl. Phys. Lett., vol. 104, p. 251907, 2014.

[15] M. Liu, H. Y. Hwang, H. Tao, A. C. Strikwerda, K. Fan, G. R. Keiser, A. J. Sternbach, K. G. West, S. Kittiwatanakul, J. Lu, S. A. Wolf, F. G. Omenetto, X. Zhang, K. A. Nelson, and R. D. Averitt, "Terahertzfield-induced insulator-to-metal transition in vanadium dioxide metamaterial.," Nature, vol. 487, no. 7407, pp. 345-8, Jul. 2012.

[16] D. Krebs, S. Raoux, C. T. Rettner, G. W. Burr, M. Salinga, and M. Wuttig, "Threshold field of phase change memory materials measured using phase change bridge devices," Appl. Phys. Lett., vol. 95, no. 8, p. 2101, 2009.

[17] T. Matsunaga, J. Akola, S. Kohara, T. Honma, K. Kobayashi, E. Ikenaga, R. O. Jones, N. Yamada, M. Takata, and R. Kojima, "From local structure to nanosecond recrystallization dynamics in AgInSbTe phasechange materials," Nat. Mater., vol. 10, no. February, p. 129, 2011.

[18] P. Zalden, A. von Hoegen, P. Landreman, M. Wuttig, and A. M. Lindenberg, "How supercooled liquid phase-change materials crystallize: Snapshots after femtosecond optical excitation," Chem. Mater., vol. 27, no. 16, p. 5641, 2015.

[19] M. A. Seo, H. R. Park, S. M. Koo, D. J. Park, J. H. Kang, O. K. Suwal, S. S. Choi, P. C. M. Planken, G. S. Park, N. K. Park, Q. H. Park, and D. S. Kim, "Terahertz field enhancement by a metallic nano slit operating beyond the skin-depth limit," Nat. Photonics, vol. 3, no. 3, pp. 152-156, 2009.

[20] See Supplemental Material [url], which includes Refs. [32-46]

[21] F. Kadlec, C. Kadlec, and P. Kužel, "Contrast in terahertz conductivity of phase-change materials," Solid State Commun., vol. 152, no. 10, pp. 852-855, 2012.

[22] W. K. Njoroge, M. Wuttig, and I. Introduction, "Crystallization kinetics of sputter-deposited amorphous AgInSbTe films,” J. Appl. Phys., vol. 90, no. 8, pp. 3816-3821, 2001.

[23] K. Shportko, S. Kremers, M. Woda, D. Lencer, J. Robertson, and M. Wuttig, "Resonant bonding in crystalline phase-change materials," Nat. Mater., vol. 7, no. 8, pp. 653-658, 2008.

[24] H. E. Swanson and E. Tagte, "Standard X-Ray Diffraction Powder Patterns," in National Bureau of Standards Circular 539, vol. I, 1953, pp. 1-96.

[25] M. Simon, M. Nardone, V. G. Karpov, and I. V. Karpov, "Conductive path formation in glasses of phase change memory," J. Appl. Phys., vol. 108, no. 6, p. 4514, 2010.

[26] G. Weiser, U. Dersch, and P. Thomas, "Polarized electroabsorption spectra of amorphous semiconductors," Philos. Mag. Part B, vol. 57, no. 6, pp. 721-735, Jun. 1988.

[27] I. V. Karpov, M. Mitra, D. Kau, G. Spadini, Y. A. Kryukov, and V. G. Karpov, "Evidence of field induced nucleation in phase change memory," Appl. Phys. Lett., vol. 92, no. 17, p. 173501, 2008.

[28] G. C. Sosso, S. Caravati, and M. Bernasconi, "Vibrational properties of crystalline $\mathrm{Sb}_{2} \mathrm{Te}_{3}$ from first principles,” J. Phys. Condens. Matter, vol. 21, no. 9, p. 095410, Mar. 2009.

[29] P. Zalden, A. von Hoegen, P. Landreman, M. Wuttig, and A. M. Lindenberg, "How supercooled liquid phase-change materials crystallize: Snapshots after femtosecond optical excitation," Chem. Mater., vol. 27, no. 16, p. $5641,2015$.

[30] J. Siegel, A. Schropp, J. Solis, C. N. Afonso, and M. Wuttig, "Rewritable phase-change optical recording in $\mathrm{Ge}_{2} \mathrm{Sb}_{2} \mathrm{Te}_{5}$ films induced by picosecond laser pulses," Appl. Phys. Lett., vol. 84, no. 13, p. 2250, 2004.

[31] M. Anbarasu, M. Wimmer, G. Bruns, M. Salinga, and M. Wuttig, "Nanosecond threshold switching of $\mathrm{GeTe}_{6}$ cells and their potential as selector devices," Appl. Phys. Lett., vol. 100, no. 14, p. 143505, 2012. 
[32] H. Y. Hwang, S. Fleischer, N. C. Brandt, B. G. Perkins, M. Liu, K. Fan, A. Sternbach, X. Zhang, R. D. Averitt, and K. A. Nelson, "A review of non-linear terahertz spectroscopy with ultrashort tabletop-laser pulses," J. Mod. Opt., vol. 62, no. 18, pp. 1447-1479, 2015.

[33] P. C. M. Planken, H.-K. Nienhuys, H. J. Bakker, and T. Wenckebach, "Measurement and calculation of the orientation dependence of terahertz pulse detection in ZnTe," J. Opt. Soc. Am. B, vol. 18, no. 3, p. 313, 2001.

[34] M. Naftaly, Ed., Terahertz Metrology. Artech House, 2015.

[35] I. Wilke and S. Sengupta, "Nonlinear Optical Techniques for Terahertz Pulse Generation and DetectionOptical Rectification and Electrooptic Sampling," in Terahertz Spectroscopy, S. Dexheimer, Ed. CRC Press, 2008.

[36] S. Casalbuoni, H. Schlarb, B. Schmidt, B. Steffen, P. Schmüser, and A. Winter, "Numerical studies on the electro-optic sampling of relativistic electron bunches," Proc. IEEE Part. Accel. Conf., vol. 2005, pp. 3070-3072, 2005.

[37] K. Strössner, S. Ves, and M. Cardona, "Refractive index of GaP and its pressure dependence," Phys. Rev. $B$, vol. 32, no. 10, pp. 6614-6619, 1985.

[38] K. Fan, H. Y. Hwang, M. Liu, A. C. Strikwerda, A. Sternbach, J. Zhang, X. Zhao, X. Zhang, K. A. Nelson, and R. D. Averitt, "Nonlinear terahertz metamaterials via field-enhanced carrier dynamics in GaAs," Phys. Rev. Lett., vol. 110, no. 21, pp. 1-5, 2013.

[39] D. J. Cook and R. M. Hochstrasser, "Intense terahertz pulses by four-wave rectification in air.," Opt. Lett., vol. 25, no. 16, pp. 1210-2, Aug. 2000.

[40] D. Grischkowsky, S. Keiding, M. van Exter, and C. Fattinger, "Far-infrared time-domain spectroscopy with terahertz beams of dielectrics and semiconductors," J. Opt. Soc. Am. B, vol. 7, no. 10, p. 2006, 1990.

[41] J. L. M. Oosthoek, D. Krebs, M. Salinga, D. J. Gravesteijn, G. A. M. Hurkx, and B. J. Kooi, “The influence of resistance drift on measurements of the activation energy of conduction for phase-change material in random access memory line cells," J. Appl. Phys., vol. 112, no. 8, p. 4506, 2012.

[42] K. Lai, W. Kundhikanjana, M. Kelly, and Z. X. Shen, "Modeling and characterization of a cantilever-based near-field scanning microwave impedance microscope," Rev. Sci. Instrum., vol. 79, no. 6, p. 3703, 2008.

[43] W. Kundhikanjana, K. Lai, H. Wang, H. Dai, M. A. Kelly, and Z. X. Shen, "Hierarchy of electronic properties of chemically derived and pristine graphene probed by microwave imaging," Nano Lett., vol. 9, no. 11, pp. 3762-3765, 2009.

[44] P. Jost, "Charge Transport in Phase-Change Materials," PhD thesis, RWTH Aachen University, 2013.

[45] S. G. Bishop, P. C. Taylor, D. L. Mitchell, and L. H. Slack, "Far infrared and microwave conductivity spectrum of semiconducting $\mathrm{Tl}_{2} \mathrm{Se} \cdot \mathrm{As}_{2} \mathrm{Te}_{3}$ glass," J. Non. Cryst. Solids, vol. 5, no. 4, pp. 351-357, 1971.

[46] N. F. Mott and E. A. Davis, Electronic processes n non-crystalline materials. Oxford: Clarendon Press, 1979. 